\title{
SÍNTESE DE AMINOÁLCOOIS DERIVADOS DO D-MANITOL\#
}

\section{Pedro S. M. de Oliveira e Vitor F. Ferreira}

Departamento de Química Orgânica, Instituto de Química, Universidade Federal Fluminense, Outeiro de S.

João Batista, s/n, 24020-150 Niterói - RJ, Brasil

Marcus Vinicius N. de Souza* e Erika Martins de Carvalho

Instituto de Tecnologia em Fármacos - Far-Manguinhos, Fundação Oswaldo Cruz, 21041-250 Rio de Janeiro - RJ, Brasil

Recebido em 4/1/08; aceito em 7/3/08; publicado na web em 9/4/08

\begin{abstract}
SYNTHESIS OF AMINOALCOHOLS FROM D-MANNITOL. Aminoalcohols have found important applications in synthetic and medicinal chemistry, being used as chiral building blocks for the synthesis of many biologically active compounds. This class of compounds has been also used as chiral auxiliaries and ligands in asymmetric synthesis. Due to the importance of aminoalcohols in the treatment of several diseases, such as tuberculosis, the aim of this article is the synthesis and preliminary evaluation against tuberculosis of six aminoalcohols in 5 or 6 steps using D-mannitol as starting material, which is a useful carbohydrate employed in many syntheses.
\end{abstract}

Keywords: aminoalcohols; mannitol; tuberculosis.

\section{INTRODUÇÃO}

Os aminoálcoois e seus derivados constituem uma importante família de compostos orgânicos de interesse farmacológico. Isto pode ser confirmado pelo grande número de novas moléculas contendo o núcleo aminoálcool, desenvolvidas para uso em diversas doenças: doença de Alzheimer, ${ }^{1}$ processos inflamatórios, ${ }^{2,3}$ esclerose múltipla ${ }^{4}$ e malária. ${ }^{5}$ Para destacar o potencial dessa classe de substâncias, podese citar o Etambutol (1), fármaco de primeira escolha no combate à tuberculose, ${ }^{6}$ e os derivados da D-galactose $\mathbf{2}$ e $\mathbf{3}$ sintetizados por De Almeida e colaboradores ${ }^{7}$ (Figura 1) que apresentam atividade biológica interessante frente ao Mycobacterium tuberculosis.

Acredita-se que aminoálcoois conjugados sejam capazes de atuarem inibindo a biossíntese da parede celular micobacteriana, ${ }^{8}$ que tem como uma de suas funções controlar o transporte de diferentes fármacos ${ }^{9}$ para as cavidades intracelulares.

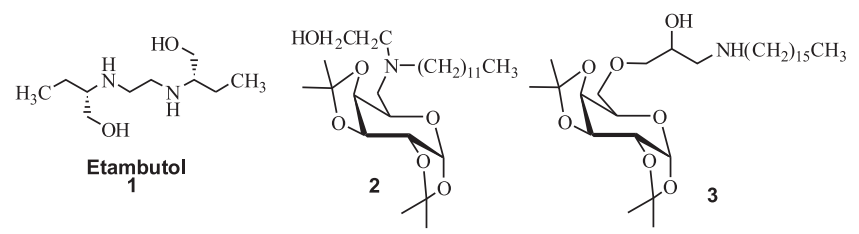

Figura 1. Alguns aminoálcoois que possuem atividade antimicobacteriana

Os carboidratos de baixos pesos moleculares são extremamente atrativos como materiais de partida em diversas sínteses devido à disponibilidade em grande quantidade, baixo custo e por serem enantiomericamente puros. ${ }^{10}$ Neste sentido se destaca o D-manitol (4) que é um carboidrato poliálcool altamente versátil e muito utilizado para os mais diversos fins. Por exemplo, o D-manitol é utilizado pela indústria farmacêutica como excipiente do tipo diluente, em comprimidos destinados a dissolverem-se na boca, devido à agradá-

"Este trabalho é dedicado à memória da inesquecível Profa. Helena Maria Carvalho Ferraz que tanto contribuiu para a Química Orgânica Sintética no Brasil e para a organização e projeção da nossa Sociedade Brasileira de Química *e-mail: marcos_souza@far.fiocruz.br vel sensação de doçura e frescor. Também é utilizado como estabilizante, pois não é higroscópico, sendo aconselhável seu uso em comprimidos contendo compostos sensíveis à umidade, tais como ácido acetilsalicílico e vitamina C. Além disso, outro fator importante é a incapacidade de ser absorvido no trato gastrointestinal. Esta propriedade torna o D-manitol um excelente diurético osmótico ${ }^{11}$ para neuroanestesia e neurorreanimação. ${ }^{12}$ Nestes casos, o D-manitol atua diminuindo o volume dos edemas cerebrais em pacientes com traumatismo craniano. ${ }^{13} \mathrm{O}$ D-manitol também é útil como agente de diagnóstico em provas de função renal e no tratamento e prevenção de falência aguda renal, reduzindo o acúmulo excessivo de fluido celular e, assim, aumentando a excreção urinária.

Do ponto de vista da síntese orgânica, o D-manitol (4) apresenta ampla aplicação, pois possui eixo de simetria $C_{2}$ e 4 centros quirais, o que o torna um excelente material de partida para síntese de blocos de construção quirais pequenos (small chiral building blocks) ${ }^{14}$ como por exemplo os acetonídeos 5-8 que são úteis na obtenção de diversos compostos enantiomericamente puros e biologicamente ativos ${ }^{15}$ (Esquema 1).

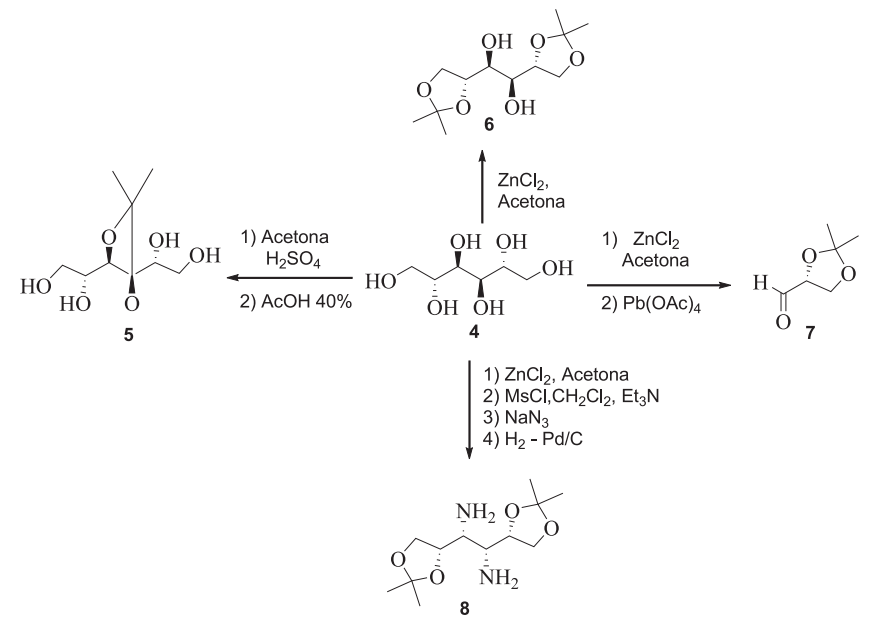

Esquema 1. Rotas de síntese para a obtenção de blocos quirais a partir do D-manitol (4) 
A urgência na descoberta de novos medicamentos para doenças negligenciadas e extremamente negligenciadas tem motivado a pesquisa e o desenvolvimento destas classes de doenças em vários países, inclusive no Brasil. ${ }^{6}$ Tendo em vista a necessidade de novos fármacos tuberculostáticos para o arsenal terapêutico,,$^{16} \mathrm{o}$ nosso grupo de pesquisa vem trabalhando intensamente nos últimos anos na síntese de novos possíveis protótipos ativos conta $M$. tuberculosis. ${ }^{17}$ Neste contexto, este trabalho teve por objetivo sintetizar aminoálcoois enatiomericamente puros a partir do D-manitol (4), como possíveis candidatos a agentes tuberculostáticos.

\section{PARTE EXPERIMENTAL}

Os espectros de RMN de ${ }^{1} \mathrm{H}$ e de ${ }^{13} \mathrm{C}$ foram obtidos em espectrômetro Bruker Avance 500 nas freqüências de 400 e 500 $\mathrm{MHz}$ (para ${ }^{1} \mathrm{H}$ ) e 100 e $125,0 \mathrm{MHz}$ (para ${ }^{13} \mathrm{C}$ ), em $\mathrm{CDCl}_{3}$, DMSO$d_{6}$ ou $\mathrm{D}_{2} \mathrm{O}$. Os deslocamentos químicos estão expressos em ppm

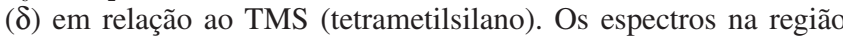
do infravermelho foram obtidos em espectrofotômetro Perkin-Elmer FT-IR, de feixe duplo, em pastilhas de $\mathrm{KBr}$ anidro ou em feixe sobre janelas de $\mathrm{NaCl}$. Os valores para as absorções estão expressos em número de onda, utilizando-se como unidade o centímetro recíproco $\left(\mathrm{cm}^{-1}\right)$. Os espectros de massa $(\mathrm{EM} / \mathrm{ES})$ foram feitos em um espectrômetro modelo Micromass ZQ Waters. O triacetonídeo 9 foi obtido a partir do D-manitol (4) conforme procedimento descrito por Le Merrer e colaboradores. ${ }^{18,20}$ As aminas foram destiladas à pressão reduzida antes serem utilizadas nas reações.

\section{3,4-Isopropilideno-D-manitol (5)}

Em um balão de fundo redondo de $500 \mathrm{~mL}$ contendo $10 \mathrm{~g}$ de triacetonídeo foram adicionados $200 \mathrm{~mL}$ de solução de ácido acético $40 \%$ (v/v). O balão foi fechado e a reação mantida sob agitação constante a $40{ }^{\circ} \mathrm{C}$ por aproximadamente $1 \mathrm{~h}$, sendo acompanhada por $\mathrm{CCF}$ (eluente $\mathrm{CHCl}_{3} / \mathrm{MeOH}$ 8:2). Após esse intervalo de tempo, o balão foi conectado a um rotaevaporador e evaporado até secura com auxílio de tolueno (aproximadamente 1,5 L) entre 45 e $50{ }^{\circ} \mathrm{C}$ obtendo-se $7 \mathrm{~g}$ de produto bruto.

\section{2,6-p-Toluenossulfonil-3,4-isopropilideno-D-manitol (10)}

Em um balão de fundo redondo de $500 \mathrm{~mL}$ contendo $7 \mathrm{~g}$ de $\mathbf{5}$ foram adicionados $40 \mathrm{~mL}$ de piridina e a solução resfriada a $-5{ }^{\circ} \mathrm{C}$ por $10 \mathrm{~min}$, seguida de adição lenta de 2,05 equivalentes de cloreto de $p$-toluenossulfonila. A reação foi mantida à $0{ }^{\circ} \mathrm{C}$ sob agitação constante por $4 \mathrm{~h}$, sendo em seguida adicionados $100 \mathrm{~mL}$ de solução gelada de ácido clorídrico $6 \mathrm{~N}$ e igual volume de éter etílico $(100 \mathrm{~mL})$ mantendo-se a agitação por 5 min $\mathrm{O}$ extrato etéreo foi separado e lavado com solução saturada de bicarbonato de sódio (100 mL), seco com sulfato de sódio e evaporado à pressão reduzi$\mathrm{da}$, fornecendo $14,5 \mathrm{~g}$ de um produto extremamente viscoso, que foi utilizado sem prévia purificação na etapa seguinte.

\section{1,6-dianidro-3,4-O-isopropilideno-D-manitol (11)}

Ao balão da etapa anterior já contendo $14,5 \mathrm{~g}$ de $\mathbf{1 0}$, foram adicionados $175 \mathrm{~mL}$ de metanol destilado. Após a solubilização do mesmo, foram adicionados $6,8 \mathrm{~g}$ (2,5 eq.) de carbonato de potássio anidro previamente seco em estufa a vácuo a $80{ }^{\circ} \mathrm{C}$ por $12 \mathrm{~h}$. A reação foi mantida sob agitação constante a temperatura ambiente por 2,5 h sendo acompanhada por CCF (eluente hexano/Et ${ }_{2} \mathrm{O}$ 1:1). Após esse intervalo, a agitação foi interrompida e o excesso de $\mathrm{K}_{2} \mathrm{CO}_{3}$ diluído com $100 \mathrm{~mL}$ de água. A fase aquosa foi extraída duas vezes com $100 \mathrm{~mL}$ de $\mathrm{CH}_{2} \mathrm{Cl}_{2}$, as fases orgânicas foram reunidas, lavadas com solução saturada de $\mathrm{NH}_{4} \mathrm{Cl}$, secas com $\mathrm{MgSO}_{4} \mathrm{e}$ evaporadas a pressão reduzida, fornecendo $8 \mathrm{~g}$ de produto bruto.
Este foi então purificado por cromatografia em coluna flash utilizando mistura de hexano-Et $2 \mathrm{O}$ 9:1. Rendimento: $1,7 \mathrm{~g}$ (17\% a partir do D-manitol em quatro etapas).

$\mathrm{RMN}$ de ${ }^{1} \mathrm{H}\left[300 \mathrm{MHz}, \mathrm{CDCl}_{3}\right]$ $\delta: 3.84$ (dd, J=3,1 e 1,4 Hz, 2H; H3 e H-4); 3,15-3,11 (m, 2H; H-2 e H-5); 2,85 (dd, J=4,7 e 4,0 Hz, 2H; H-1a e H-6a ou H-1b e H-6b); 2,73 (dd, J=4,7 e 2,6 Hz, 2H; H1a e H-6a ou H-1b e H-6b); 1,45 (s; 6H; H-8) ppm; RMN de ${ }^{13} \mathrm{C}$ $\left(75 \mathrm{MHz}, \mathrm{CDCl}_{3}\right.$ ) $\delta: 110,2$ (C-7); 78,1 (C-3 e C-4); 51,3 (C-2 e C5); 44,9 (C-1 e C-6); 26,5 (C-8).

\section{Procedimento geral para a preparação dos aminoálcoois trissubstituídos (12a-c)}

À uma solução de 1,6-dianidro-3,4-O-isopropilideno-D-manitol 11 (100 mg, 0,54 mmols) em isopropanol ( $3 \mathrm{~mL}$ ) foram adicionados lentamente 2 equivalentes da amina apropriada, e a mistura aquecida a $80-85^{\circ} \mathrm{C}$ entre 3,5 a $6 \mathrm{~h}$. A reação foi acompanhada por CCF utilizando o eluente hexano/éter etílico 1:1. Após o consumo do reagente, o solvente e o excesso da amina foram removidos por destilação a pressão reduzida fornecendo o produto desejado.

\section{1,6-didesoxi-1,6-di-piperidinil-3,4-O-isopropilideno-D-manitol (12a)}

Este produto foi obtido sob a forma de sólido amarelo pálido em 95\% (182 mg) de rendimento utilizando-se Piperidina. p.f.: 72$74{ }^{\circ} \mathrm{C}$; IV (cm-1 filme): 3369; 2985; 2934; 2855; 2797; $1070 \mathrm{~cm}^{-1}$, $\mathrm{RMN}$ de ${ }^{1} \mathrm{H}[400,00 \mathrm{MHz}$ (FIDRES $\left.\pm 0,73 \mathrm{~Hz}), \mathrm{CDCl}_{3}\right] \delta: 3,85$ (dd, $J=4,9$ e 1,6 Hz, 2H, H-3 e H-4); 3,81-3,75 (m, 2H, H-2 e H-5); 2,59 (dd, $J=12,6$ e 3,3 Hz, 6H, H-1a e H-6a ou H-1b e H-6b e H-1'a ou H-1'b); 2,42-2,34 (m, 4H, H-1'a ou H-1'b); 2,46 (dd, J=12,6 e 9,7 Hz, H-1a e H-6a ou H-1b e H-6b); 1,64-1,53 (m, 8H, H-2'); 1,49-1,41 (m, 4H, H-3'); 1,37 (s, 6H, H-8) ppm; RMN de ${ }^{13} \mathrm{C}(100,0$ $\left.\mathrm{MHz}, \mathrm{CDCl}_{3}\right) \delta:$ 109,6 (C-7); 81,6 (C-3 e C-4); 68,0 (C-2 e C-5); 61,4 (C-1 e C-6); 55,0 (C-1'); 27,3 (C-8); 26,1 (C-2'); 24,3 (C-3') ppm; EM/ESI: $m / z,[\mathrm{M}+\mathrm{H}]^{+}:$: 357,6 (massa esperada: 357,28).

\section{1,6-didesoxi-1,6-di-N,N-dipropilamino-3,4-O-isopropilideno-D- manitol (12b)}

Este produto foi obtido sob a forma de óleo amarelo em $91 \%$ (191 mg) de rendimento utilizando-se $N, N$-di-Propilamina. IV ( $\mathrm{cm}^{-1}$; filme): $3411 ; 2960 ; 2874 ; 2814 ; 1461 ; 1379 ; 1243 ; 1215 ; 1068$ $\mathrm{cm}^{-1}, \mathrm{RMN}$ de ${ }^{1} \mathrm{H}\left[500,00 \mathrm{MHz}\right.$ (FIDRES $\pm 0,16 \mathrm{~Hz}$ ), $\left.\mathrm{CDCl}_{3}\right] \delta: 3,89$ (dd, $J=5,4$ e 1,3 Hz, 2H, H-3 e H-4); 3,71-3,65 (m, 2H, H-2 e H-5); 2,65 (dd, $J=10,0$ e 2,4 Hz, 2H, H-1a e'H-6a ou H-1b e H-6b); 2,572,46 (m, 6H, H-1a e H-6a ou H-1b e H-6b e H-1'a ou H-1'b); 2,432,38 (m, 4H, H-1'a ou H-1'b); 1,54-1,40 (m, 8H, H-2'); 1,39 (s, 6H, $\mathrm{H}-8) ; 0,87$ (t, $\left.J=6,0 \mathrm{~Hz}, 12 \mathrm{H}, \mathrm{H}-3^{\prime}\right)$ ppm; RMN de ${ }^{13} \mathrm{C}(125,0 \mathrm{MHz}$, $\mathrm{CDCl}_{3}$ ) $\delta: 109,3$ (C-7); 81,5 (C-3 e C-4); 68,2 (C-2 e C-5); 57,1 (C1 e C-6); 56,2 (C-1'); 27,2 (C-8); 20,3 (C-2'); 11,8 (C-3') ppm; EM/ ESI: $m / z,[\mathrm{M}+\mathrm{H}]^{+}:$: 389,6 (massa esperada: 389,34).

\section{1,6-didesoxi-1,6-di-N,N-dibutilamino-3,4-O-isopropilideno-D- manitol (12c)}

Este produto foi obtido sob a forma de óleo amarelo claro em $80 \%$ (192 mg) de rendimento utilizando-se N,N-di-Butilamina. IV ( $\mathrm{cm}^{-1}$; filme): 3413; 2957; 2933; 2873; 1462; 1379; 1070; RMN de ${ }^{1} \mathrm{H}[400,00 \mathrm{MHz}$ (FIDRES $\left.\pm 0,13 \mathrm{~Hz}), \mathrm{CDCl}_{3}\right] \delta: 3,90(\mathrm{dd}, J=4,8 \mathrm{e}$ 1,5 Hz, 2H, H-3 e H-4); 3,71-3,64 (m, 2H, H-2 e H-5); 2,64 (dd, J= 12,8 e 3,5 Hz, 2H, H-1a e H-6a ou H-1b e H-6b); 2,59-2,48 (m, 6H, H-1a e H-6a ou H-1b e H-6b e H-1'a ou H-1'b); 2,46-2,37 (m, 4H, H-1'a ou H-1'b); 1,47-1,41 (m, 8H, H-2'); 1,39 (s, 6H, H-8); 1,361,21 (m, 8H, H-3'); 0,90 (t, J= 7,3 Hz, 12H, H-4') ppm; RMN de ${ }^{13} \mathrm{C}\left(125,0 \mathrm{MHz}, \mathrm{CDCl}_{3}\right)$ \&: 109,3 (C-7); 81,5 (C-3 e C-4); 68,2 (C2 e C-5); 57,1 (C-1 е C-6); 54,1 (C-1'); 29,3 (C-2'); 27,2 (C-8); 
20,6 (C-3'); 14,0 (C-4') ppm; EM/ESI: $m / z$ [M+H] $]^{+}:$445,4 (massa esperada: 445,40$)$.

Procedimento geral para a preparação dos aminoálcoois dissubstituídos (12d-g)

Ao balão reacional contendo 1,6-di-anidro-3,4-O-isopropilidenoD-manitol (11) (100 mg, 0,54 mmols) foram adicionados lentamente $3 \mathrm{~mL}$ da amina apropriada, e a mistura foi mantida à temperatura ambiente por 12-30 h. A reação foi acompanhada por CCF. utilizando o eluente hexano/éter etílico 1:1. Após esse intervalo de tempo a agitação foi interrompida e o excesso de amina removido por destilação à pressão reduzida, fornecendo o produto em alto grau de pureza.

\section{1,6-didesoxi-1,6-di-N-isopropilamino-3,4-O-isopropilideno-D- manitol (12d)}

Este produto foi obtido sob a forma de sólido branco em $95 \%$ (156 mg) de rendimento utilizando-se Isopropilamina. p.f.: 86-88 ${ }^{\circ} \mathrm{C}$; IV (cm ${ }^{-1}$; filme): 3306; 2968; 1647; 1470; 1382; 1069; RMN de ${ }^{1} \mathrm{H}[400,00 \mathrm{MHz}$ (FIDRES $\left.\pm 0,13 \mathrm{~Hz}), \mathrm{CDCl}_{3}\right] \delta: 3,88(\mathrm{dd}, J=5,6 \mathrm{e}$ 1,8 Hz, 2H, H-3 e H-4); 3,73-3,68 (m, 2H, H-2 e H-5); 2,96 (dd, J= 12,0 e 3,6 Hz, 2H, H-1a e H-6a ou H-1b e H-6b); 2,79 (sept, $J=6,4$ Hz, 2H, H-1'); 1,38 (s, 6H, H-8); 1,08 (d, J=6,4 Hz, 6H, H-2' ou H3'); 1,07 (d, J=6,4 Hz, 6H, H-2' ou H-3') ppm; RMN de ${ }^{13} \mathrm{C}(100,0$ $\mathrm{MHz}, \mathrm{CDCl}_{3}$ ) $\delta: 108,8$ (C-7); 81,4 (C-3 e C-4); 71,0 (C-2 e C-5); 50,1 (C-1 e C-6); 48,9 (C-1'); 26,9 (C-8); 23,1 (C-2' ou C-3'); 22,8 (C-2' ou C- 3'); EM/ESI: $m / z$ [M+H] ${ }^{+}:$: 305,4 (massa esperada: $305,24)$.

\section{1,6-didesoxi-1,6-di-N-propilamino-3,4-O-isopropilideno-D- manitol (12e)}

Este produto foi obtido sob a forma de sólido amarelo claro em 97\% (159 mg) de rendimento utilizando-se Propilamina. p.f. 46$48{ }^{\circ} \mathrm{C}$; IV (cm ${ }^{-1}$; filme): 3308; 2961; 2934; 1462; $1071 \mathrm{~cm}^{-1}, \mathrm{RMN}$ de ${ }^{1} \mathrm{H}\left[500,00 \mathrm{MHz}\right.$ (FIDRES $\pm 0,16 \mathrm{~Hz}$ ), $\left.\mathrm{CDCl}_{3}\right] \delta: 3,90$ (dd, $J=$ 5,4 e 1,6 Hz, 2H, H-3 e H-4); 3,87-3,74 (m, 2H, H-2 e H-5); 2,99 (dd, $J=12,2$ e 3,2 Hz, 2H, H-1a e H-6a ou H-1b e H-6b); 2,76 (dd, $J=12,2$ e 7,2 Hz, 2H, H-1a e H-6a ou H-1b e H-6b); 2,64 (dt, $J=$ 7,2 e 3,2 Hz, 4H, H-1'); 1,57 (sext, J= 7,2 Hz, 4H, H-2'); 1,38 (s, $6 \mathrm{H}, \mathrm{H}-8) ; 0,93$ (t, $J=7,2 \mathrm{~Hz}, 6 \mathrm{H}, \mathrm{H}-3$ ') ppm; RMN de ${ }^{13} \mathrm{C}(125,0$ $\mathrm{MHz}, \mathrm{CDCl}_{3}$ ) $\delta:$ 109,1 (C-7); 81,3 (C-3 e C-4); 70,6 (C-2 e C-5); 52,3 (C-1 e C-6); 51,6(C-1'); 26,9 (C-8); 22,7 (C-2'); 11,6 (C3')ppm; EM/ESI: $m / z,[\mathrm{M}+\mathrm{H}]^{+}:$305,5 (massa esperada: 305,24).

\section{1,6-didesoxi-1,6-di-N-terc-butilamino-3,4-O-isopropilideno-D- manitol (12f)}

Este produto foi obtido sob a forma de sólido branco em $95 \%$ $\left(170 \mathrm{mg}\right.$ ) de rendimento utilizando-se $t$-Butilamina. p.f.: $87-89{ }^{\circ} \mathrm{C}$; IV (cm ${ }^{-1}$; filme): $3297 ; 2968 ; 1650 ; 1480 ; 1368 ; 1216 ; 1071$; RMN de ${ }^{1} \mathrm{H}\left[400,00 \mathrm{MHz}(\right.$ FIDRES $\left.\pm 0,13 \mathrm{~Hz}), \mathrm{CDCl}_{3}\right] \delta: 3,88(\mathrm{dd}, J=$ 5,3 e $1,7 \mathrm{~Hz}, 2 \mathrm{H}, \mathrm{H}-3$ e H-4); 3,64-3,71 (m, 2H, H-2 e H-5); 2,93 (dd; $J=11,6 ; J=3,8 \mathrm{~Hz} ; 2 \mathrm{H}, \mathrm{H}-1$ a e H-6a ou H-1b e H-6b); 2,62 (dd; $J=11,6 \mathrm{~Hz}, J=7,0 \mathrm{~Hz}, 2 \mathrm{H}, \mathrm{H}-1 \mathrm{a}$ e H-6a ou H-1b e H-6b); 1,38 (s, 6H, H-8); 1,11 (s, 18H, H-2') ppm; RMN de ${ }^{13} \mathrm{C}(100,0$ $\mathrm{MHz}, \mathrm{CDCl}_{3}$ ) $\delta: 108,8$ (C-7); 81,5 (C-3 e C-4); 71,2 (C-2 e C-5); 50,3 (C-1') ; 45,4 (C-1 e C-6); 29,0 (C-2'); 26,9 (C-8) ppm; EM/ ESI: $m / z,[\mathrm{M}+\mathrm{H}]^{+.}: 333,4$ (massa esperada: 333,28 ).

\section{1,6-didesoxi-1,6-di-N-butilamino-3,4-O-isopropilideno-D-manitol (12g)}

Este produto foi obtido sob a forma de óleo amarelo em $80 \%$ (144 mg) de rendimento utilizando-se Butilamina. IV ( $\mathrm{cm}^{-1}$; filme): 3306 ; 2932; 1463; 1380; 1251; $1071 \mathrm{~cm}^{-1}$, RMN de ${ }^{1} \mathrm{H}[400,00 \mathrm{MHz}$ $($ FIDRES $\left.\pm 0,16 \mathrm{~Hz}), \mathrm{CDCl}_{3}\right] \delta: 3,88(\mathrm{dd}, J=5,4$ e $1,6 \mathrm{~Hz}, 2 \mathrm{H}, \mathrm{H}-3$ e H-4); 3,77-3,70 (m, 2H, H-2 e H-5); 2,93 (dd, $J=12,0$ e 3,7 Hz, 2H, H-1a e H-6a ou H-1b e H-6b); 2,69 (dd, $J=12,0$ e 6,9 Hz, 2H, H-1a e H-6a ou H-1b e H-6b); 2,66-2,58 (m, 4H, H-1'); 1,48 (quint, $J=7,3 \mathrm{~Hz}, 4 \mathrm{H}, \mathrm{H}-2$ '); 1,39-1,31 (m, 4H, H-3'); 1,37 (s, 6H, H-8); 0,92 (t, $J=7,3 \mathrm{~Hz}, 6 \mathrm{H}, \mathrm{H}-4$ ') ppm; RMN de ${ }^{13} \mathrm{C}\left(100,0 \mathrm{MHz}, \mathrm{CDCl}_{3}\right)$


49,7 (C-1'); 32,1 (C-2'); 27,0 (C-8); 20,6 (C-3'); 14,1 (C-4') ppm; EM/ESI: $m / z,[\mathrm{M}+\mathrm{H}]^{+}:$: 333,4 (massa esperada: 333,28).

\section{Procedimento geral para a obtenção dos aminoálcoois (13a-c $\boldsymbol{e}$ 13d-g)}

Uma mistura de 13a-d ou 13e-h (50 mg) com uma solução de $\mathrm{HCl} 0,5 \mathrm{~N}$ (1 mL) foi agitada por $12 \mathrm{~h}$ sendo acompanhada por $\mathrm{CCF}$, utilizando a mistura $\mathrm{CHCl}_{3}-\mathrm{MeOH}$ (8:2) como eluente. Após esse intervalo de tempo a água e o ácido foram removidos sob pressão reduzida com auxílio de uma bomba de alto vácuo e aquecimento a $40{ }^{\circ} \mathrm{C}$, fornecendo um resíduo mais polar que o material de partida.

\section{1,6-didesoxi-1,6-di-piperidinil-D-manitol (13a)}

Este produto foi obtido sob a forma de sólido verde claro em rendimento quantitativo. p.f.: $164{ }^{\circ} \mathrm{C}$; IV $\left(\mathrm{cm}^{-1} ; \mathrm{KBr}\right): 3306,2944$, 2855, 2727, 1627, 1459, 1405, 1095, 1076, 1030 $\mathrm{cm}^{-1}$; RMN de ${ }^{1} \mathrm{H}$ [400,00 MHz (FIDRES $\left.\pm 0,13 \mathrm{~Hz}), \mathrm{D}_{2} \mathrm{O}\right] \delta: 4,15(\mathrm{t}, J=8,4 \mathrm{~Hz}, 2 \mathrm{H}$, H-2 e H-5); 3,76 (d, $J=8,4$ Hz, 2H, H-3 e H-4); 3,62 (d, $J=11,6$ $\mathrm{Hz}, 2 \mathrm{H}, \mathrm{H}-1$ 'b ou H-1'b); 3,54 (d, $J=11,7 \mathrm{~Hz}, 2 \mathrm{H}, \mathrm{H}-1$ 'a ou H1"a); 3,47 (d, $J=13,3$ Hz, 2H, H-1a e H-6a ou H-1b e H-6b); 3,20 (t, $J=12 \mathrm{~Hz}, 2 \mathrm{H}, \mathrm{H}-1$ a e H-6a ou H-1b e H-6b) 3,09 (t, $J=11,3 \mathrm{~Hz}$, 2H, H-1'a ou H-1"a); 2,96 (t, $J=11,3$ Hz, 2H, H-1'b ou H-1"'b); 1,99-1,88 (m, 4H, H-2'a ou H-2'a ou H-2'b ou H-2'b); 1,85-1,69 (m, 6H, H-2'a ou H-2"'a ou H-2'b ou H-2'b e H-3'a ou H-3'b); 1,59-1,51 (m, 2H, H-3'a ou H-3'b) ppm; RMN de ${ }^{13} \mathrm{C}(100,0 \mathrm{MHz}$, $\left.\mathrm{D}_{2} \mathrm{O}\right) \delta:$ 70,5 (C-3 e C-4); 64,6 (C-2 e C-5); 59,6 (C-1 e C-6); 55,2 e 52,2(C-1' e C-1"); 22,4 e 22,3(C-2' ou C-2"); 21,0 (C-3') ppm; EM/ESI: $m / z,[\mathrm{M}+\mathrm{H}]^{+.}: 317,5$ (massa esperada: 317,24 ).

\section{1,6-didesoxi-1,6-di-N,N-dipropilamino-D-manitol (13b)}

Este produto foi obtido sob a forma de óleo castanho em rendimento quantitativo. IV ( $\left.\mathrm{cm}^{-1} ; \mathrm{KBr}\right)$ : 3351, 2973, 2883, 1638, 1473, $1073,754 \mathrm{~cm}^{-1}, \mathrm{RMN}^{1} \mathrm{H}[400,00 \mathrm{MHz}($ FIDRES $\pm 0,13 \mathrm{~Hz})$, $\left.\mathrm{DMSO}_{d-6}\right] \delta: 5,70(\mathrm{~d}, J=6,3 \mathrm{~Hz}, 2 \mathrm{H}, \mathrm{OH}) ; 5,02(\mathrm{~d}, J=7,8 \mathrm{~Hz}, 2 \mathrm{H}$, $\mathrm{OH})$; 3,95-3,90 (m, 2H, H-2 e H-5); 3,54 (t, $J=7,9$ Hz, 2H, H-3 e H-4); 3,39-3,36 (m, 2H, H-1a e H-6a ou H-1b e H-6b); 3,13-2,85 (m, 10H, H-1a e H-6a ou H-1b e H-6b e H-1' e H-1" ); 1,78-1,62 (m, 8H, H-2' e H-2"); 0,88 (t, $J=7,2 \mathrm{~Hz}, 12 \mathrm{H}, \mathrm{H}-3$ ' e H-3") ppm; RMN ${ }^{13} \mathrm{C}\left(100,0 \mathrm{MHz}, \mathrm{DMSO}_{d-6}\right) \delta: 70,5$ (C-3 e C-4); 64,8 (C-2 e C-5); 56,4 (C-1 e C-6); 55,1 e 53,9 (C-1' ou C-1"); 16,6 ou 16,2 (C2' ou C-2'); 10,8 (C-3') ppm; EM/ESI: $m / z[\mathrm{M}+\mathrm{H}]^{+.}$: 349,5 (massa esperada: 349,31$)$.

\section{1,6-didesoxi-1,6-di-N,N-dibutilamino-D-manitol (13c)}

Este produto foi obtido sob a forma de óleo amarelo em rendimento quantitativo. IV ( $\left.\mathrm{cm}^{-1} ; \mathrm{KBr}\right)$ : 3338, 2962, 2875, 1638, 1468, $1070,738 \mathrm{~cm}^{-1}, \mathrm{RMN}^{1} \mathrm{H}$ [400,00 MHz (FIDRES $\pm 0,13 \mathrm{~Hz}$ ), D $\mathrm{D}_{2} \mathrm{O}$ $\delta$ : 4,08 (td, $J=9,3$ e 2,5 Hz; $2 \mathrm{H}, \mathrm{H}-2$ e H-5); 3,75 (d, $J=8,1 \mathrm{~Hz}$, $2 \mathrm{H}, \mathrm{H}-3$ e H-4); 3,52 (dd, $J=8,1$ e $2,5 \mathrm{~Hz} ; 2 \mathrm{H}, \mathrm{H}-1$ a e H-6a ou H$1 \mathrm{~b}$ e H-6b); 3,32-3, 18 (m, 10H, H-1a e H-6a ou H-1b e H-6b e H-1' e H-1"); 1,79-1,62 (m, 8H, H-2' e H-2"); 1,38 (sext, $J=7,4 \mathrm{~Hz}$, $8 \mathrm{H}, \mathrm{H}-3$ ' e H-3"); 0,93 (t, J=7,4 Hz, 12H, H-4' e H-4") ppm; RMN ${ }^{13} \mathrm{C}\left(100,0 \mathrm{MHz}, \mathrm{D}_{2} \mathrm{O}\right) \delta: 70,7$ (C-3 e C-4); 65,1 (C-2 e C-5); 56,0 (C-1 e C-6); 52,6 (C-1'); 25,2 e 24,7 (C-2' e C-2'); 19,3 (C-3'); 12,8 (C-4') ppm; EM/ESI: $m / z$ [M+H] ${ }^{+.}$: 405,6 (massa esperada: $405,37)$. 


\section{1,6-didesoxi-1,6-di-N-isopropilamino-D-manitol (13d)}

Este produto foi obtido sob a forma de sólido branco em rendimento quantitativo. p.f.: $181-183{ }^{\circ} \mathrm{C}$; IV $\left(\mathrm{cm}^{-1}\right.$; $\left.\mathrm{KBr}\right): 3412,2978$, 1600, 1399, 1070, 918, $745 \mathrm{~cm}^{-1}$; RMN ${ }^{1} \mathrm{H}$ [400,00 MHz (FIDRES $\left.\pm 0,13 \mathrm{~Hz}), \mathrm{D}_{2} \mathrm{O}\right] \delta: 3,99(\mathrm{dt}, J=8,7$ e $2,1 \mathrm{~Hz}, 2 \mathrm{H}, \mathrm{H}-2$ e H-5); 3,77 (d, $J=8,7 \mathrm{~Hz}, 2 \mathrm{H}, \mathrm{H}-3$ e H-4); 3,44 (dd, $J=13,0$ e 2,3 Hz, 2H, H1a e H-6a ou H-1b e H-6b); 3,13 (dd, $J=13,0$ e 10,1 Hz, 2H, H-1a e H-6a ou H-1b e H-6b); 3,08 (t, $J=7,9$ Hz, 4H, H-1'); 1,74 (sext., $J=7,4 \mathrm{~Hz}, 4 \mathrm{H}, \mathrm{H}-2$ '); 0,98 (t, $J=7,4 \mathrm{~Hz}, 6 \mathrm{H}, \mathrm{H}-3$ ') ppm; RMN ${ }^{13} \mathrm{C}$

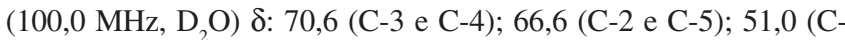
1'); 47,6 (C-1 e C-6); 18,4 (C-2' ou C-2"); 17,9 (C-2' ou C-2") ppm; EM/ESI: $m / z,[\mathrm{M}+\mathrm{H}]^{+} .:$265,5 (massa esperada: 265,21).

\section{1,6-didesoxi-1,6-di-N-propilamino-D-manitol (13e)}

Este produto foi obtido sob a forma de sólido amarelo claro em rendimento quantitativo. p.f.: $175^{\circ} \mathrm{C}$ (dec.); IV ( $\mathrm{cm}^{-1}$; KBr): 3368 , 2964, 2790, 2539, 2432, 1597, 1461, 1412, 1073, $759 \mathrm{~cm}^{-1}$; RMN ${ }^{1} \mathrm{H}\left[400,00 \mathrm{MHz}(\right.$ FIDRES $\left.\pm 0,12 \mathrm{~Hz}), \mathrm{D}_{2} \mathrm{O}\right] \delta: 3,99(\mathrm{dt}, J=8,7 \mathrm{e}$ $2,1 \mathrm{~Hz}, 2 \mathrm{H}, \mathrm{H}-2$ e H-5); 3,77 (d, $J=8,7 \mathrm{~Hz}, 2 \mathrm{H}, \mathrm{H}-3$ e H-4); 3,44 (dd, $J=13,0$ e 2,3 Hz, 2H, H-1a e H-6a ou H-1b e H-6b); 3,13 (dd, $J=13,0$ e $10,1 \mathrm{~Hz}, 2 \mathrm{H}, \mathrm{H}-1 \mathrm{a}$ e H-6a ou H-1b e H-6b); 3,08 (t, $J=$ 7,9 Hz, 4H, H-1'); 1,74 (sext., $J=7,4$ Hz, 4H, H-2'); 0,98 (t, $J=7,4$ $\left.\mathrm{Hz}, 6 \mathrm{H}, \mathrm{H}-3^{\prime}\right)$ ppm; RMN de ${ }^{13} \mathrm{C}\left(100,0 \mathrm{MHz}, \mathrm{D}_{2} \mathrm{O}\right) \delta: 73,4$ (C-3 e C-4); 69,2 (C-2 e C-5); 53,2 (C-1 e C-6); 52,3 (C-1'); 21,8 (C-2'); 13,0 (C-3') EM/ESI: $m / z,[\mathrm{M}+\mathrm{H}]^{+.:}$265,4 (massa esperada: 265,21).

\section{1,6-didesoxi-1,6-di-N-terc-butilamino-D-manitol (13f}

Este produto foi obtido sob a forma de sólido branco em rendimento quantitativo. p.f.: $175-177{ }^{\circ} \mathrm{C}$; IV $\left(\mathrm{cm}^{-1}\right.$; $\left.\mathrm{KBr}\right)$ : 3338,2978 , 2799, 2485, 1614, 1378, 1069, 1064, $758 \mathrm{~cm}^{-1}$; RMN ${ }^{1} \mathrm{H}[400,00$ $\mathrm{MHz}$ (FIDRES $\left.\pm 0,13 \mathrm{~Hz}), \mathrm{D}_{2} \mathrm{O}\right] \delta: 3,90(\mathrm{t}, J=7,4 \mathrm{~Hz}, 2 \mathrm{H}, \mathrm{H}-2 \mathrm{e}$ H-5); 3,77 (d, $J=8,2 \mathrm{~Hz}, 2 \mathrm{H}, \mathrm{H}-3$ e H-4); 3,42 (d, $J=11,1 \mathrm{~Hz}, 2 \mathrm{H}$, H-1a e H-6a ou H-1b e H-6b); 3,07 (t, $J=11,2$ Hz, 2H, H-1a e H$6 \mathrm{a}$ ou H-1b e H-6b); 1,39 (s, 18H, H-2') ppm; RMN ${ }^{13} \mathrm{C}(100,0$ $\left.\mathrm{MHz}, \mathrm{D}_{2} \mathrm{O}\right) \delta$ : 70,6 (C-3 e C-4); 67,0 (C-2 e C-5); 57,5 (C-1'); 44,7 (C-1 e C-6); 24,8 (C-2') ppm; EM/ESI: $m / z$ [M+H] ${ }^{+.:}$293,5 (massa esperada: 293,24).

\section{1,6-didesoxi-1,6-di-N-butilamino-D-manitol (13g)}

Este produto foi obtido sob a forma de sólido branco em rendimento quantitativo. p.f.: $266-268{ }^{\circ} \mathrm{C}$ (dec.); IV ( $\mathrm{cm}^{-1}$; $\mathrm{KBr}$ ): 3351 , 2963, 2874, 2798, 2442, 1596, 1462, 1411, 1074, $741 \mathrm{~cm}^{-1}$; RMN de ${ }^{1} \mathrm{H}\left[400,00 \mathrm{MHz}\right.$ (FIDRES $\pm 0,13 \mathrm{~Hz}$ ), $\left.\mathrm{D}_{2} \mathrm{O}\right] \delta: 3,99$ (dt, $J=9,6$ e $2,8 \mathrm{~Hz}, 2 \mathrm{H}, \mathrm{H}-2$ e H-5); 3,77 (d, $J=8,3 \mathrm{~Hz}, 2 \mathrm{H}, \mathrm{H}-3$ e H-4); 3,44 (dd; $J=13,0$; e 2,9 Hz; 2H, H-1a e H-6a ou H-1b e H-6b); 3,163,09 (m, 6H, H-1a e H-6a ou H-1b e H-6b e H-1'); 1,70 (quint., $J=$ 7,4 Hz, 4H, H-2'); 1,41(sext., $J=7,4$ Hz, 4H, H-3'); 0,94 (t, $J=$ $7,4 \mathrm{~Hz}, 6 \mathrm{H}, \mathrm{H}-4$ ') ppm; RMN de ${ }^{13} \mathrm{C}\left(100,0 \mathrm{MHz}, \mathrm{D}_{2} \mathrm{O}\right) \delta: 70,6(\mathrm{C} 3$ e C-4); 66,3 (C-2 e C-5); 50,3 (C-1 e C-6); 47,7 (C-1'); 27,4 (C2'); 19,2 (C-3'); 12,8 (C-4'); EM/ESI: $m / z$ [M+H]+.: 293,5 (massa esperada: 293,24).

\section{Avaliação antimicrobiana}

Para a realização dos ensaios da atividade antimicrobiana dos compostos 12c, 12f, 13a, 13c, 13f e 13g foram utilizadas microplacas estéreis com 96 orifícios, preparadas de modo que cada orifício apresenta um total de $200 \mu \mathrm{L}$ de uma mistura composta do meio de cultura apropriado, do composto a ser testado e da suspensão bacteriana e utilizou-se como padrão de comparação a rifampicina que apresenta um MIC igual 1,0 $\mu \mathrm{g} / \mathrm{mL}$.

Após 5 dias de incubação, foram adicionados em cada orifício $15 \mu \mathrm{L}$ de Alamar Blue ${ }^{\circledR}$ e as microplacas foram incubadas por mais
$24 \mathrm{~h}$ a $37^{\circ} \mathrm{C}$. Terminado este tempo, foi observada a mudança de coloração em cada orifício, sendo o MIC definido como a menor concentração do composto que delimita a mudança de coloração de azul para rósea.

\section{RESULTADOS E DISCUSSÃO}

A metodologia empregada para a síntese dos aminoálcoois desejados utilizou o intermediário bis-epóxido 11, obtido a partir do Dmanitol (4) em quatro etapas num rendimento global de $17 \%$, utilizando a rota sintética de Le Merrer e colaboradores (Esquema 2). ${ }^{18}$

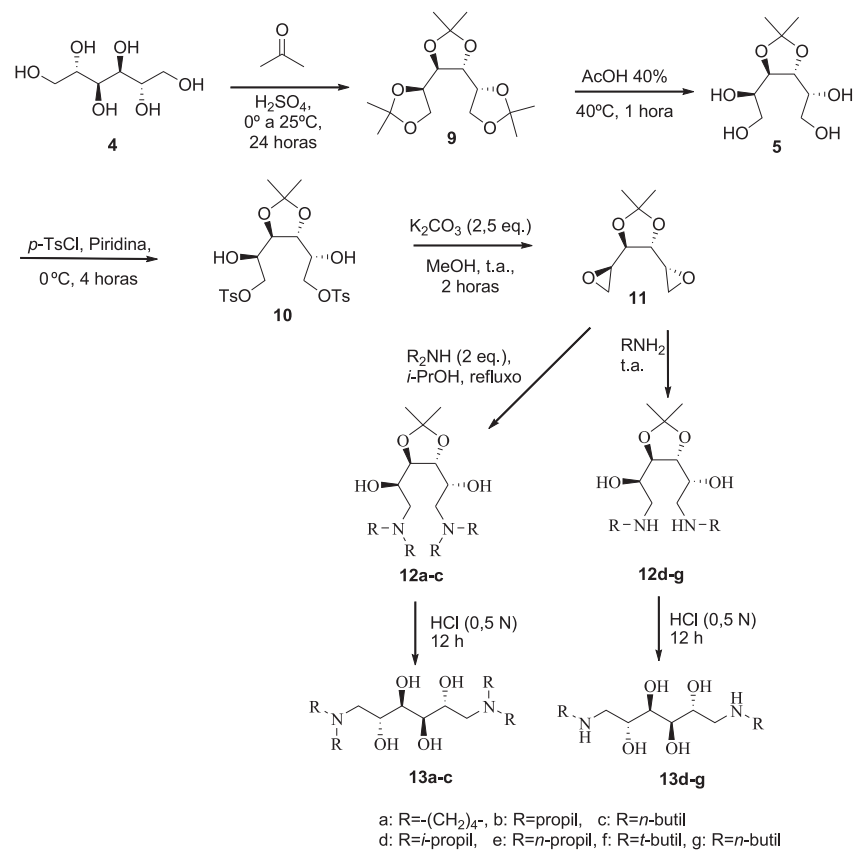

Esquema 2. Rota sintética utilizada para a preparação dos aminálcoois 13 e 14

Primeiramente, realizou-se a proteção das hidroxilas obtendose o triacetonídeo 9 que, através da desproteção seletiva das hidroxilas primárias, levou à formação do mono-acetonídeo 5. Devese ressaltar que, para o sucesso dessa reação, é fundamental que a evaporação do ácido acético seja realizada numa temperatura entre 45-50 ${ }^{\circ} \mathrm{C}$ e com sucessivas adições de tolueno. Um tempo prolongado de exposição ao ácido e uma temperatura elevada favorecem a total desproteção de $\mathbf{5}$, retornando ao material de partida. O material obtido nesta etapa está suficientemente puro para que se proceda à próxima etapa de tosilação regiosseletiva das hidroxilas primárias, levando ao bis-tosilato 10. Nesta etapa, uma pequena quantidade de piridina, apenas o suficiente para solubilizar o monoacetonídeo, foi utilizada a fim de evitar a exposição prolongada e possível hidrólise do grupo isopropilideno no momento de extração da mesma com solução de $\mathrm{HCl} 6 \mathrm{~N}$. Devido à instabilidade de 11 em meio básico, ${ }^{19}$ utilizou-se 2,5 equivalentes de $\mathrm{K}_{2} \mathrm{CO}_{3}$ anidro a fim de evitar a sua degradação. Além disso, a purificação do produto foi feita por cromatografia em coluna utilizando como eluente uma mistura de hexano e éter etílico para facilitar a evaporação dos solventes e evitar perda do produto 11 (Esquema 2).

Considerando que as aminas secundárias são menos reativas devido ao maior volume estérico de substituintes e que esta reação, quando realizada em solventes polares próticos, é lenta e pode levar até 4 dias,${ }^{20}$ decidiu-se fazer as reações de obtenção dos derivados 12a-c sob refluxo usando 2 equivalentes da amina apropriada em isopropanol. Através desta metodologia obteve-se 12a e os 
aminoálcoois inéditos $\mathbf{1 2 b}$ e $\mathbf{1 2 c}$ com rendimentos que variaram entre $80-95 \%$ e tempo reacional reduzido, entre 3,5 a $6 \mathrm{~h}$. A síntese dos derivados aminados dissubstituídos inéditos 12d-g foi realizada à temperatura ambiente, utilizando as aminas apropriadas como solvente, obtendo-se os produtos com rendimentos que variaram entre 80-97\%. Buscando produtos solúveis em água o isopropilideno foi hidrolisado utilizando-se uma solução de ácido diluída $(\mathrm{HCl}$ $0,5 \mathrm{~N}$ ) fornecendo 13a e os aminoalcoóis inéditos $\mathbf{1 3 b} \mathbf{b}$-g em rendimento quantitativo. Todas as substâncias tiveram suas estruturas confirmadas utilizando-se técnicas de espectroscopia.

A atividade antimicrobiana dos compostos 12c, 12f, 13a, 13c, 13f e 13g foi avaliada, sendo o composto 12c o único que se mostrou capaz de inibir o crescimento do $M$. tuberculosis a uma concentração mínima inibitória (MIC) de $50 \mu \mathrm{g} / \mathrm{mL}$, indicando que a presença de grupos lipofílicos, ligados ao nitrogênio e/ou oxigênio é importante para a atividade antibacteriana desta classe de compostos.

\section{CONCLUSÃO}

Neste trabalho foram sintetizados em cinco ou seis etapas quatorze aminoálcoois dos quais doze ainda não foram descritos na literatura. Foram ainda realizados testes contra o M. tuberculosis H37Rv ATCC n 27294 dos aminoálcoois 12c, 12f, 13a, 13c, 13f e 13g. Resultados preliminares indicam a ausência de atividade tuberculostática para os aminoálcoois 12f, 13a, 13c, 13f e 13g, no entanto o composto protegido $12 \mathrm{c}$ do aminoálcool $13 \mathbf{c}$ apresentou um MIC $=50 \mu \mathrm{g} / \mathrm{mL}$, indicando que a funcionalização dos grupos hidroxilas e/ou aminas pode ser importante para a atividade biológica. Os demais aminoálcoois protegidos, 12a, 12b, 12d, 12e, 12g, 13b, 13d e 13e, encontram-se em fase de avaliação, bem como se encontra em andamento um estudo da relação estrutura atividade e a síntese de novas substâncias desta classe de compostos.

\section{AGRADECIMENTOS}

Ao CNPq, à CAPES, FAPERJ, FINEP, Far-Manguinhos FIOCRUZ e UFF pelas bolsas e apoio financeiro às pesquisas.

\section{REFERÊNCIAS E NOTAS}

1. Maillard, M.; Tucker, J. A.; WO 02/100820 A1 2002; Herold, P.; Stutz, S.; EP 1745776 Al 2007.

2. Bräunlich, G.; Es-Sayed, M.; Fischer, R.; Fugmann, B.; Henning, R.; Schneider, S.; Sperzel, M.; Schlemmer, K. H.; Sturton, G.; Fitzgerald, M.; Briggs, B.; Conception, A.; Bullock, W.; WO 00/69841 2000.

3. Berger, M.; Schmees, N.; Schaecke, H.; Baeurle, S.; Rehwinkel, H.; Mengel, A.; Krolikiewicz, K.; Grossbach, D.; Voigtlaender, D.; WO 2006/ 066950 A2 2006.

4. Albert, R.; Cooke, N. G.; Nuesslein-Hildesheim, B.; Weiler, S.; WO 2007/ 0288212007.
5. Howarth, J.; Lloyd, D. G.; J. Antimicrob. Chemother. 2000, 46, 625.

6. http://www.who.int/tb/en/, acessada em Dezembro 2007; De Souza, M. V. N.; Recent Pat. Anti-Infect. Drug Discov. 2006, 1, 33; De Souza, M. V. N.; Curr. Opin. Pulm. Med. 2006, 12, 167.

7. De Almeida, M. V.; Taveira, A. F.; Le Hyriac, M.; Reis, E. F. C.; Araújo, D. P.; Ferreira, A. P.; De Souza, M. A.; Alves, L. L.; Lourenço, M. C. S.; Vicente, F. R. C.; Bioorg. Med. Chem. 2007, 15, 7789; De Almeida, M . A.; Le Hyriac, M.; Amarante, G. W.; Lourenço, M. C. S.; Brandão, M. L. L.; Eur. J. Med. Chem. 2007, 42, 1076.

8. Pathak, A. K.; Pathak, V.; Seitz, L.; Maddry, J. A.; Gurcha, S. S.; Besra, G. S.; Suling, W. J.; Reynolds, R. C.; Bioorg. Med. Chem. 2001, 9, 3129; Pathak, A. K.; Pathak, V.; Suling, W. J.; Gurcha, S. S.; Morehouse, C. B.; Besra, G. S.; Maddry, J. A.; Reynolds, R. C.; Bioorg. Med. Chem. 2002, 10, 923; Wen, X.; Crick, D. C.; Brennan, P. J.; Hultin, P. G.; Bioorg. Med. Chem. 2003, 11, 3579; Maddry, J. A.; Bansal, N.; Bermudez, L. E.; Comber, R. N.; Orme, I. M.; Suling, W. J.; Bioorg. Med. Chem. Lett. 1998, 8, 237; Tripathi, R. P.; Tripathi, R.; Tiwari, V. K.; Bala, L.; Sinha, S.; Srivastava, A.; Srivastava, R.; Srivastava, B. S.; Eur. J. Med. Chem. 2002, 37, 773; Tewari, N.; Tiwari, V. K.; Mishra, R. C.; Tripathi, R. P.; Srivastava, A. K.; Ahemad, R.; Srivastava, R.; Srivastava, B. S.; Bioorg. Med. Chem. 2003, 11, 2911; Tewari, N.; Tiwari, V. K.; Tripathi, R. P.; Gaikwad, A.; Sinha, S.; Shukla, P. K.; Srivastava, R.; Srivastava, B. S.; Bioorg. Med. Chem. Lett. 2004, 14, 329; Katiyar, D.; Tiwari, V. K.; Tewari, N.; Verma, S. S.; Sinha, S.; Gaikwad, A.; Srivastava, A.; Chaturvedi, V.; Srivastava, R.; Srivastava, B. S.; Tripathi, R. P.; Eur. J. Med. Chem. 2005, 40, 351; De Souza, A. O.; Pedrosa, M. T.; Alderete, J. B.; Cruz, A. F.; Prado, M. A.; Alves, R. B.; Silva, C. L.; Pharmazie 2005, 60, 396.

9. Katz, A. H.; Caufield, C. E.; Curr. Pharm. Des. 2003, 9, 857.

10. Ferreira, V. F.; Quim. Nova 1995, 18, 267.

11. A forma medicamentosa do D-manitol é conhecida como Osmitrol® e, em geral, é uma solução aquosa entre 15 a $25 \%$.

12. Archer, D. P.; Freymond, D.; Ravussin, P.; Ann. Françaises Anesth. Reanim. 1995, 14, 77 .

13. Duchateau, F. X.; Burnod, A.; Chollet, C.; Ricard Hibon; Samain, E.; Marty, J.; Ann. Françaises Anesth. Reanim. 2004, 23, 879.

14. Takano, S.; Kurotaki, M.; Takahashi, M.; Ogasawara, K.; Synthesis 1986, 5,403 .

15. De March, P., Figueredo, M. ; Font, J. ; Raya, J.; Org. Lett. 2000, 2, 163; Kagawa, N.; Ihara, M.; Toyota, M.; Org. Lett. 2006, 8, 875; Young, I. S.; Kerr, M. A.; J. Am. Chem. Soc. 2007, 129, 1465.

16. Souza, M. V. N.; Vasconcelos, T. A.; Cardoso, S. H.; Almeida, M. V.; Curr. Med. Chem. 2006, 13, 455.

17. Lourenço, M. C. S.; Junior, I. N.; Souza, M. V. N.; Med. Mal. Infect. 2007, 37, 295; Pinheiro A. C.; Kaiser, C. R.; Lourenço, M. C. S.; Souza, M. V. N.; Wardell, J. L.; Wardell, S. M. S. V.; J. Chem. Res. Syn. 2007, 3, 182; Gallardo, H.; Conte, G.; Bryk, F.; Lourenço, M. C. S.; Costa, M. S.; Ferreira, V. F.; J. Braz. Chem. Soc. 2007, 18, 1285; Costa, M. S.; Boechat, N.; Rangel, E. A.; Lourenço, M. C. S.; Junior, I. N.; Castro, H. C.; Souza, A. M. T.; Silva, F. C.; Wardell, S. M. S. V.; Rodrigues, C. R.; Ferreira, V. F.; Bioorg. Med. Chem. 2006, 14, 8644; Souza, M. V. N.; Junior, I. N.; Miranda, G. B. P.; Lourenço, M. C. S.; Vasconcelos, T. A.; Pais, K. C.; Wardell, J. L.; Wardell, S. M. S. V.; Junior, J. P. A.; Lett. Drug Design Discov. 2006, 3, 424.

18. Le Merrer, Y.; Dureault, A.; Greck, C.; Micas-Languin, D.; Gravier, C.; Depezay, J.; Heterocycles 1987, 25, 541.

19. Nugiel, D. A.; Jacobs, K.; Tabaka, C. A.; Teleha, C. A.; Org. Synth. 2005, 81,140 .

20. Mc Cort, I.; Sanière, M.; Le Merrer, Y.; Tetrahedron 2003, 59, 2693; Le Merrer, Y.; Sanière, M.; McCort, I.; Dupuy, C.; Depezay, J. C.; Tetrahedron Lett. 2001, 42, 2661. 